\title{
ON DERIVATIVES OF ORTHOGONAL POLYNOMIALS. II ${ }^{1}$
}

\author{
H. L. KRALL
}

It has been shown $[1,2,7]^{2}$ that if $\left\{y_{n}(x)\right\}$ is a set of orthogonal polynomials whose derivatives $\left\{y_{n}^{\prime}(x)\right\}$ also form an orthogonal set, then the original set $\left\{y_{n}(x)\right\}$ are either Jacobi, Laguerre, or Hermite polynomials. In the papers referred to, the ordinary definition of orthogonal polynomials was used, so of course the result was based on this definition. Since then, however, there has appeared $[3,5]^{3}$ a definition of generalized orthogonal polynomials ${ }^{4}$ and it is our purpose here to find what G.O.P. sets have G.O.P. derivatives. Besides the classical polynomials, we shall find another class of polynomials which have this property. Of course, this new class will be G.O.P., but not ordinary orthogonal polynomials.

Definition. Given a set of constants $\left\{c_{n}\right\}$ subject to the condition

$$
\Delta_{n+1}=\left|\begin{array}{cccc}
c_{0} & c_{1} & \cdots & c_{n} \\
c_{1} & c_{2} & \cdots & c_{n+1} \\
\cdot & \cdot & \cdots & c_{n} \\
c_{n} & c_{n+1} & \cdots & c_{2 n}
\end{array}\right| \neq 0, \quad n=0,1, \cdots
$$

the polynomials

$$
y_{n}(x)=\left|\begin{array}{lll}
c_{0} & c_{1} \cdots c_{n} \\
c_{1} & c_{2} \cdots c_{n+1} \\
\cdot & \cdots \cdots \\
c_{n-1} & c_{n} \cdots c_{2 n-1} \\
1 & x \cdots x^{n}
\end{array}\right|
$$

form a set of G.O.P.

It has been shown $[5,4]$ that there exists a function of bounded variation $\psi(x)$ (hence infinitely many), such that

\footnotetext{
${ }^{1}$ Presented to the Society, April 26, 1940, under the title Orthogonal polynomial solutions of a certain fourth order differential equation.

2 The numbers in square brackets refer to the bibliography at the end.

${ }^{3}$ So far as this author knows, the idea of generalized orthogonal polynomials originated with I. M. Sheffer, who suggested its use in [3].

${ }^{4}$ Hereafter we shall use the abbreviation G.O.P. for generalized orthogonal polynomials.
} 


$$
c_{n}=\int_{-\infty}^{+\infty} x^{n} d \psi(x) .
$$

If the constants $\left\{c_{n}\right\}$ are real and $\Delta_{n}>0$ for all values of $n$, this definition becomes the usual definition of orthogonal polynomials. In this case one of the $\psi(x)$ will be monotonically non-decreasing.

In this paper we follow the method of Hahn [1] who showed that if $\left\{y_{n}(x)\right\}$ and $\left\{y_{n}^{\prime}(x)\right\}$ are sets of orthogonal polynomials, the set $\left\{y_{n}(x)\right\}$ satisfies a differential equation of the form ${ }^{5}$

$$
\left(l_{22} x^{2}+l_{21} x+l_{20}\right) y_{n}^{\prime \prime}(x)+\left(l_{11} x+l_{10}\right) y_{n}^{\prime}(x)=\lambda_{n} y_{n}(x)
$$

where the $\left\{l_{i j}\right\}$ are constants and $\lambda_{n}=l_{11} n+l_{22} n(n-1)$. Hahn divided his discussion of (1) according to the roots of the coefficient of $y_{n}^{\prime \prime}(x)$. Following his example and making suitable linear transformations, we find that there are four distinct cases of (1) to be considered:

$$
\begin{aligned}
x(1-x) y_{n}^{\prime \prime}(x)+\{\alpha-(\alpha+\beta) x\} y_{n}^{\prime}(x) & \\
+n(\alpha+\beta+n-1) y_{n}(x) & =0, \\
x y_{n}^{\prime \prime}(x)+(\alpha-h x) y_{n}^{\prime}(x)+h n y_{n}(x) & =0, \\
y_{n}^{\prime \prime}(x)-2 x y_{n}^{\prime}(x)+2 n y_{n}(x) & =0, \\
x^{2} y_{n}^{\prime \prime}(x)+(k x+l) y_{n}^{\prime}(x)-n(k+n-1) y_{n}(x) & =0 .
\end{aligned}
$$

By using the ordinary definition, it readily follows that the only orthogonal polynomial solutions of these equations are the Jacobi polynomials (with $\alpha, \beta>0$ ) from (2), the Laguerre polynomials (with $\alpha>0$ ) from (3), and the Hermite polynomials from (4).

We now examine these differential equations for G.O.P. solutions. For this discussion, we need a result from [3]:

In order that there exists a set of G.O.P. as solutions of (1), it is necessary and sufficient that the "moments" $\left\{c_{n}\right\}$ satisfy the conditions

$$
\begin{array}{r}
\left\{l_{11}+l_{22}(n-1)\right\} c_{n}+\left\{l_{10}+l_{21}(n-1)\right\} c_{n-1}+l_{20}(n-1) c_{n-2}=0, \\
n=1,2, \cdots, \\
\Delta_{n+1} \neq 0, \quad n=0,1,2, \cdots .
\end{array}
$$

Discussion of (2). Here, the recurrence relation (6) becomes $(n+\alpha+\beta-1) c_{n}-(n+\alpha-1) c_{n-1}=0, n=1,2, \cdots$. From this we get

${ }^{5}$ This part of Hahn's work applies to G.O.P. His notation has been changed to conform to [3]. 


$$
\begin{aligned}
c_{n} & =\frac{\Gamma(\alpha+n) \Gamma(\alpha+\beta) c_{0}}{\Gamma(\alpha+\beta+n) \Gamma(\alpha)}, \\
\Delta_{n+1} & =\prod_{i=0}^{n} \frac{\Gamma(i+1) \Gamma(\alpha+i) \Gamma(\beta+i) \Gamma(\alpha+\beta) c_{0}}{\Gamma(\alpha+\beta+i+n) \Gamma(\alpha) \Gamma(\beta)} .
\end{aligned}
$$

Accordingly, if $\alpha, \beta, \alpha+\beta \neq 0,-1,-2, \cdots$, the differential equation (3) has a set of G.O.P. as solutions. However, it does not seem to be easy to write out the weight function when either $\alpha$ or $\beta$ is less than zero. These polynomials have been discussed by Szegö [6].

Discussion of (3). In this case we have

$$
\begin{gathered}
h c_{n}-(n+\alpha-1) c_{n-1}=0, \\
c_{n}=\frac{\Gamma(\alpha+n) c_{0}}{h^{n} \Gamma(\alpha)}, \quad \Delta_{n+1}=\prod_{i=0}^{n} \frac{\Gamma(i+1) \Gamma(\alpha+i) c_{0}}{h^{2 i} \Gamma(\alpha)} .
\end{gathered}
$$

Accordingly, if $h \neq 0$, and $\alpha \neq 0,-1,-2, \cdots$, the differential equation (3) has a set of G.O.P. as solutions. These polynomials have also been discussed by Szegö [6].

Discussion of (4). There are no exceptional cases here. The solutions are Hermite polynomials.

Discussion of (5). In this case we have

$$
\begin{gathered}
(k+n-1) c_{n}+l c_{n-1}=0, \\
c_{n}=\frac{l^{n} \Gamma(k) c_{0}}{\Gamma(k+n)}, \quad \Delta_{n+1}=\prod_{i=0}^{n} \frac{\left(-l^{2}\right)^{i} \Gamma(k) \Gamma(i+1) c_{0}}{\Gamma(k+i+n)} .
\end{gathered}
$$

Accordingly, if $l \neq 0$, and $k \neq 0,-1,-2, \cdots$, the differential equation (5) has a set of G.O.P. as solutions. For no value of $k$ will $\Delta_{n+1}$ be greater than zero for all values of $n$. These polynomials were discussed by Hahn [1] to the extent of showing that they are not orthogonal polynomials in the ordinary sense. They are not mentioned by any of the other authors.

Returning to (1) and differentiating, we get

$$
\begin{aligned}
\left(l_{22} x^{2}+l_{21} x+l_{20}\right) y_{n}^{\prime \prime \prime}(x)+\left\{\left(l_{11}+2 l_{22}\right) x+l_{10}\right. & \left.+l_{21}\right\} y_{n}^{\prime \prime}(x) \\
& =\left(\lambda_{n}-l_{11}\right) y_{n}^{\prime}(x) .
\end{aligned}
$$

Thus, if a set of polynomials $\left\{y_{n}(x)\right\}$ satisfies (1), the set of derivatives $\left\{y_{n}^{\prime}(x)\right\}$ satisfies the equation

$$
\begin{aligned}
\left(l_{22} x^{2}+l_{21} x+l_{20}\right) \phi^{\prime \prime}(x)+\left\{\left(l_{11}+2 l_{22}\right) x+l_{10}+\right. & \left.l_{21}\right\} \phi^{\prime}(x) \\
& =\left(\lambda_{n}-l_{11}\right) \phi(x) .
\end{aligned}
$$


Since (7) is the same type as (1) and since (7) will not be an exceptional case if (1) is not, it follows that the only sets of G.O.P. whose derivatives are again G.O.P. are:

(i) the Jacobi polynomials with $\alpha, \beta, \alpha+\beta \neq 0,-1,-2, \cdots$,

(ii) the Laguerre polynomials with $h \neq 0$ and $\alpha \neq 0,-1,-2, \cdots$,

(iii) the Hermite polynomials,

(iv) the polynomial solutions of (5) with $l \neq 0$, and $k \neq 0,-1$, $-2, \cdots$.

\section{BIBLIOGRAPHY}

1. W. Hahn, Über die Jacobischen Polynome und zwei verwandte Polynomklassen, Mathematische Zeitschrift, vol. 39 (1935), pp. 634-638.

2. H. L. Krall, On derivatives of orthogonal polynomials, this Bulletin, vol. 42 (1936), pp. 423-428.

3. - Certain differential equations for Tchebycheff polynomials, Duke Mathematical Journal, vol. 4 (1938), pp. 705-718.

4. G. Pólya, Sur l'indétermination d'un problème voisin du problème des moments, Comptes Rendus, de l'Académie des Sciences, Paris, vol. 207 (1938), pp. 708-711.

5. J. Shohat, Sur les polynomes orthogonaux généralisés, ibid., vol. 207 (1938), pp. 556-558.

6. G. Szegö, Orthogonal Polynomials, American Mathematical Society Colloquium Publications, vol. 23, 1939.

7. M. S. Webster, Orthogonal polynomials with orthogonal derivatives, this Bulletin, vol. 44 (1938), pp. 880-887.

Pennsyluania State College 